\title{
Two new Micarea species (Pilocarpaceae) from Western Europe
}

\author{
Pieter P. G. van den Boom ${ }^{1,2^{*}}$, Beata Guzow-Krzemińska ${ }^{3}$ \& Martin Kukwa ${ }^{3}$
}

\section{Article info}

Received: 4 Sept. 2019

Revision received: 8 Nov. 2019

Accepted: 28 Nov. 2019

Published: 2 Jun. 2020

\section{Associate Editor}

Paul Diederich

\begin{abstract}
Two new Micarea species, M. minuta and M. pseudotsugae, are described from the Netherlands based on morphological, chemical and molecular data. Micarea minuta belongs to the $M$. denigrata group and can be distinguished from other similar species of this group by its small apothecia, Sedifolia-grey pigment in the epihymenium, mostly 1-septate and small ascospores, $40-80 \mu \mathrm{m}$ wide mesopycnidia, and the lack of secondary lichen metabolites detectable by thin-layer chromatography. Micarea pseudotsugae is a member of the $M$. prasina group and is characterized by an areolate, granular to subsquamulose thallus, the presence of methoxymicareic acid, the lack of Sedifolia-grey pigment, the presence of crystals (visible in polarized light) in the apothecia only, and one type of conidia (mesoconidia). The phylogenetic position of the newly described species was studied based on mtSSU rDNA marker analysis; $M$. minuta was found to be closely related to $M$. denigrata, M. nitschkeana and $M$. subnigrata, while $M$. pseudotsugae was found to be closely related to $M$. byssacea and M. laeta. Notes on secondary chemistry, ecology, distribution and phylogeny are given.
\end{abstract}

Key words: Ascomycota, corticolous species, Lecanorales, lichens, molecular phylogenetics, secondary chemistry, taxonomy

\section{Introduction}

The lichen genus Micarea has recently been studied intensively, and several new species have been recognized worldwide (Aptroot \& Cáceres 2014; Brand et al. 2014; van den Boom \& Ertz 2014; Guzow-Krzemińska et al. 2016, 2019a; van den Boom et al. 2017a, b; 2018; Kantvilas 2018; Launis \& Myllys 2019; Launis et al. 2019a, b). Two more new Micarea species from Western Europe (Belgium and the Netherlands) are described below. Micarea minuta belongs to the Micarea denigrata group, and M. pseudotsugae belongs to the M. prasina group. The former is a corticolous species with very small apothecia, so far known from a few localities in the Netherlands but abundantly present at the type locality. The latter is a species from soft bark of Pseudotsuga trees and soft wood of a Pseudotsuga stump, so far known only from a few localities in the Netherlands and one locality in Belgium.

\footnotetext{
${ }^{1}$ Arafura 16, NL-5691 JA Son, The Netherlands

2 Evolution and Conservation Biology, University of Liège, Sart Tilman B22, B-4000 Liège, Belgium

${ }^{3}$ Department of Plant Taxonomy and Nature Conservation, Faculty of Biology, University of Gdańsk, Wita Stwosza 59, 80-308 Gdańsk, Poland

* Corresponding author e-mail: pvdboom@kpnmail.nl
}

\section{Materials and methods}

Morphological and chemical analyses of specimens

Material of the two new species was collected by the first author in the Netherlands except for one collection made in Belgium, all deposited in LG, UGDA (holotypes) and the private herbarium of the first author. The specimens used for microscopic examination were apothecial sections and squashed thallus preparations in tap water, with or without the addition of C (commercial bleach), $\mathrm{K}$ (water solution of potassium hydroxide), $\mathrm{N}$ (nitric acid) and Lugol's reagent (I) with (K/I) or without (I) pre-treatment with K. Most microscopic measurements were made in water, except those of paraphyses, which were studied in water with the addition of $\mathrm{K}$ (Orange et al. 2001). In each collection, $\sim 10$ ascospores, conidia and paraphyses were measured to $0.5 \mu \mathrm{m}$ accuracy. Chemical compounds were studied by thin-layer chromatography (TLC) using standard methods (Orange et al. 2001). The crystalline granules were observed with a compound microscope under polarized light (Launis et al. 2019a, b). 
DNA extraction, PCR amplification and DNA sequencing

DNA was extracted directly from pieces of thalli by a modified CTAB method (Guzow-Krzemińska \& Węgrzyn 2000). DNA extracts were used for PCR amplification; $25 \mu \mathrm{l}$ PCR mix contained 1U DreamTaq polymerase (Thermo Scientific) and $1 \mathrm{X}$ concentrated buffer, $0.2 \mathrm{mM}$ of each of the four dNTP's, $0.5 \mu \mathrm{M}$ of each mrSSU1 and mrSSU3R primer (Zoller et al. 1999) and 10-50 ng of genomic DNA. Amplifications were performed in a Mastercycler (Eppendorf) under conditions described in Guzow-Krzemińska et al. (2019b).

Subsequently, PCR products were treated with a mixture of 20 units of Exonuclease I and 2 units of FastAPTM Thermosensitive Alkaline Phosphatase enzymes (Thermo Scientific) at $37^{\circ} \mathrm{C}$ for $15 \mathrm{~min}$, followed by incubation at $85^{\circ} \mathrm{C}$ for $15 \mathrm{~min}$ to completely inactivate both enzymes. Sequencing of each PCR product was performed in Macrogen (www.macrogen.com) using the PCR primers.

\section{Sequence alignment and phylogenetic analysis}

The newly generated mtSSU sequences were compared with the sequences available in the GenBank database (http://www.ncbi.nlm.nih.gov/BLAST/) using BLASTn search (Altschul et al. 1990) to confirm their identity. The sequences of each marker were aligned with sequences of representatives of the genus Micarea obtained from GenBank and with Byssolecania variabilis and Byssoloma leucoblepharum as outgroup (specimens and GenBank accession numbers are listed in Table 1); 193 sequences in total were analyzed. Alignment was performed using the Guidance 2 server employing the MAFFT algorithm (Landan \& Graur 2008; Penn et al. 2010; Sela et al. 2015). Terminal ends and portions of the alignment with ambiguous positions that might not have been homologous were excluded using Gblocks 0.91b (Castresana 2000; Dereeper et al. 2008) with less stringent settings (i.e. allowing smaller final blocks, gap positions within the final blocks, and less strict flanking positions). The final alignment consisted of 193 sequences of 770 sites.

The data were analyzed using a Bayesian approach (MCMC) in MrBayes 3.2 (Huelsenbeck \& Ronquist 2001; Ronquist \& Huelsenbeck 2003) and the GTR model. Two parallel runs were performed, each using four independent chains and 10 million generations, sampling trees every 1,000th generation. Posterior probabilities (PP) were determined by calculating a majority-rule consensus tree generated from the 15,002 post-burn-in trees of the 20,002 trees sampled by the two MCMC runs using the sumt option of MrBayes.

Maximum likelihood analysis was performed using RaxML HPC v. 8 on XSEDE (Stamatakis 2014) under the GTRGAMMAI model on the CIPRES Science Gateway (Miller et al. 2010). Rapid bootstrap analyses were performed with 1,000 bootstrap replicates (BS). The RAxML tree did not contradict the Bayesian tree topology for the strongly supported branches. Therefore only the maximum likelihood tree is shown, with the posterior probabilities
(PP) of the Bayesian analysis and the bootstrap support values added near the internal branches. $\mathrm{BS} \geq 70$ and $\mathrm{PP} \geq 0.95$ were considered significant. Phylogenetic trees were visualized using FigTree v. 1.4.2 (Rambaut 2012) and the clades for previously described taxa are collapsed to reduce the size of the tree.

\section{Results and discussion}

The final DNA alignment consisted of $190 \mathrm{mtSSU}$ rDNA sequences obtained from GenBank and three sequences of this marker from the newly described species and a specimen of $M$. microsorediata. Since the topologies from the maximum likelihood and Bayesian analyses did not show any strongly supported conflict, the Bayesian tree (Harmonic mean: -13673.39) is presented in Figure 1, with added posterior probabilities and bootstrap support from maximum likelihood analysis (Final ML Optimization Likelihood: -12102.250356).

The phylogenetic reconstruction (Fig. 1) shows that Micarea forms two main lineages: a highly supported M. prasina group and allied species (1 PP, $98 \mathrm{BS}$ ), and a clade consisting of several other Micarea species (1 PP). The Micarea prasina group seems to be well studied, while the other species need further attention.

Although the M. prasina group has been widely studied recently (Guzow-Krzemińska et al. 2019a; Launis et al. 2019a, b) and numerous new species have already been recognized, here we describe another new species belonging to this group. Figure 1 shows that the entire M. prasina group is highly supported and monophyletic (1 PP, $95 \mathrm{BS}$ ) and agrees with previous phylogenies based on a mtSSU marker (e.g., Czarnota \& Guzow-Krzemińska 2010; Guzow-Krzemińska et al. 2016) or three loci (Guzow-Krzemińska et al. 2019a; Launis \& Myllys 2019; Launis et al. 2019a, b). Within the M. prasina group, two main lineages are further distinguished: the $M$. micrococca clade ( $1 \mathrm{PP}, 94 \mathrm{BS}$ ) and the $M$. prasina clade (0.99 PP) (sensu Guzow-Krzemińska et al. 2019a) with the sequences of $M$. pusilla and M. tomentosa forming a highly supported lineage (1 PP, $97 \mathrm{BS}$ ) basal to the two clades, and $M$. hedlundii and $M$. xanthonica basal to the M. micrococca clade (Fig. 1). The highly supported M. micrococca clade consists mostly of species containing methoxymicareic acid as shown by Guzow-Krzemińska et al. (2019a) and Launis et al. (2019a, b). This group accommodates the newly described $M$. pseudotsugae, which also produces methoxymicareic acid, and this species is closely related to $M$. byssacea and M. laeta (1 PP, $100 \mathrm{BS}$ ).

The M. prasina clade (sensu Guzow-Krzemińska et al. 2019a) consists mostly of species containing micareic acid and accommodates $M$. aeruginoprasina, $M$. azorica, M. falax, M. fennica, M. flavoleprosa, M. herbarum, M. isidioprasina, M. melanobola, M. meridionalis, M. nowakii, M. prasina, M. soralifera and $M$. subviridescens (Fig. 1). Moreover, M. pusilla and M. tomentosa are closely related and both belong to $M$. prasina group and are basal to the M. micrococca and M. prasina clades (sensu Guzow-Krzemińska et al. 2019a). 
Table 1. List of Micarea specimens and outgroup taxa used in this study, with their GenBank accession numbers. Sequences newly generated for this study are bolded. If the taxonomic classification of the specimen was verified in further study, two or more references are cited for a single sample.

\begin{tabular}{|c|c|c|c|c|}
\hline Species & Country/region & Voucher & Reference & mtSSU \\
\hline $\begin{array}{l}\text { Byssoloma } \\
\text { leucoblepharum }\end{array}$ & Portugal & Ekman $3502(\mathrm{BG})$ & Andersen \& Ekman 2005 & AY567778 \\
\hline Byssolecania variabilis & Costa Rica & Lücking 16033b (BG) & Andersen \& Ekman 2005 & AY567780 \\
\hline Micarea adnata & Norway & Andersen 48 (BG) & Andersen \& Ekman 2005 & AY567751 \\
\hline Micarea adnata & France & Sérusiaux s.n., DNA 3438 (LG) & van den Boom et al. 2017 & KX459344 \\
\hline Micarea aeruginoprasina & Portugal, Azores & van den Boom 51445, DNA 3973 (LG) & Guzow-Krzemińska et al. 2019a & MK562024 \\
\hline Micarea alabastrites & Norway & Andersen $17(\mathrm{BG})$ & Andersen \& Ekman 2005 & AY 567764 \\
\hline Micarea assimilata & Sweden & Kanz \& Printzen 5449 (herb. Printzen) & Andersen \& Ekman 2005 & AY567739 \\
\hline Micarea azorica & Portugal, Azores & van den Boom 51360, DNA 3980 (LG) & Guzow-Krzemińska et al. 2019a & MK562028 \\
\hline Micarea azorica & Portugal, Azores & van den Boom 51330, DNA 3976 (LG) & Guzow-Krzemińska et al. 2019a & MK562025 \\
\hline Micarea azorica & Portugal, Azores & van den Boom 51468 DNA 3977 (LG) & Guzow-Krzemińska et al. 2019a & MK562026 \\
\hline Micarea azorica & Portugal, Azores & van den Boom 51733, DNA 3978 (LG) & Guzow-Krzemińska et al. 2019a & MK562027 \\
\hline Micarea botryoides & Norway & Andersen $79 b(B G)$ & Andersen \& Ekman 2005 & AY567741 \\
\hline Micarea byssacea & Norway & Andersen 34 (BG) & $\begin{array}{l}\text { Andersen \& Ekman 2005, Czarnota } \\
\text { \& Guzow-Krzemińska } 2010\end{array}$ & AY567749 \\
\hline Micarea byssacea & Poland & Czarnota $4751(\mathrm{GPN})$ & Czarnota \& Guzow-Krzemińska 2010 & EF453664 \\
\hline Micarea byssacea & Estonia & Czarnota 4781 (GPN) & Czarnota \& Guzow-Krzemińska 2010 & EF453670 \\
\hline Micarea byssacea & Estonia & Czarnota $3956(\mathrm{GPN})$ & Czarnota \& Guzow-Krzemińska 2010 & EF453690 \\
\hline Micarea byssacea & Germany & $\begin{array}{l}\text { van den Boom, 50037, LG DNA } 3495 \\
\text { (herb. van den Boom) }\end{array}$ & van den Boom et al. 2017 & KX459345 \\
\hline Micarea byssacea & Finland & Launis 289103 DNA A98 $(\mathrm{H})$ & Launis et al. $2019 b$ & MG707768 \\
\hline Micarea byssacea & Finland & Launis 289102, DNA A97 $(\mathrm{H})$ & Launis et al. $2019 b$ & MG707769 \\
\hline Micarea byssacea & Finland & Launis 289101, DNA A96 (H) & Launis et al. $2019 b$ & MG707770 \\
\hline Micarea cinerea & Norway & Tønsberg $28572(\mathrm{BG})$ & Andersen \& Ekman 2005 & AY567763 \\
\hline Micarea clavopycnidiata & USA & Tønsberg 27215 (BG) & Andersen \& Ekman 2005 & AY567747 \\
\hline Micarea coppinsii & Norway & Tønsberg 26075 (BG) & Andersen \& Ekman 2005 & AY567761 \\
\hline Micarea czarnotae & Poland & Czarnota 4059 (GPN) & $\begin{array}{l}\text { Czarnota \& Guzow-Krzemińska 2010, } \\
\text { Launis et al. 2019b }\end{array}$ & EF453663 \\
\hline Micarea czarnotae & Poland & Czarnota 3632 (GPN) & $\begin{array}{l}\text { Czarnota \& Guzow-Krzemińska } 2010 \text {, } \\
\text { Launis et al. 2019b }\end{array}$ & EF453668 \\
\hline Micarea czarnotae & Poland & Czarnota 3179 (GPN) & $\begin{array}{l}\text { Czarnota \& Guzow-Krzemińska } 2010, \\
\text { Launis et al. } 2019 \text { b }\end{array}$ & EF453674 \\
\hline Micarea czarnotae & Poland & Czarnota 4179 (GPN) & $\begin{array}{l}\text { Czarnota \& Guzow-Krzemińska } 2010, \\
\text { Launis et al. } 2019 \text { b }\end{array}$ & EF453691 \\
\hline Micarea czarnotae & Finland & Launis 109111, DNA A604 (H) & Launis et al. $2019 b$ & MG707759 \\
\hline Micarea czarnotae & Finland & Launis 1010133, DNA A455 (H) & Launis et al. $2019 \mathrm{~b}$ & MG707760 \\
\hline Micarea czarnotae & Belgium & van den Boom 50312, DNA 3712 (LG) & Launis et al. 2019b & MG707761 \\
\hline Micarea deminuta & Bohemia & Palice \& Voriskova 6745 (herb. Palice) & Andersen 2004 & AY756446 \\
\hline Micarea denigrata & Sweden & Koffman 5 (herb. Koffman) & Andersen \& Ekman 2005 & AY567759 \\
\hline Micarea denigrata & Poland & Czarnota $4593(\mathrm{GPN})$ & Czarnota \& Guzow-Krzemińska 2010 & EF453681 \\
\hline Micarea denigrata & Netherlands & $\begin{array}{l}\text { Brand } 63258 \text { (herb. Brand), DNA } 3851 \\
\text { (LG) }\end{array}$ & van den Boom et al. 2017 & $\mathrm{KX} 459346$ \\
\hline Micarea denigrata & Germany & Sérusiaux s.n., DNA 4381, (LG) & van den Boom et al. 2017 & KX459347 \\
\hline Micarea denigrata & USA & Bjork 13019 (UBC), AFTOL-ID 4923 & Miadlikowska et al. 2014 & KJ766437 \\
\hline Micarea doliiformis & UK, Wales & Orange s.n. (herb. Orange) LG, NMH & Sérusiaux et al. 2010 & GU138666 \\
\hline Micarea doliiformis & $\begin{array}{l}\text { Canary Islands, } \\
\text { Tenerife }\end{array}$ & $\begin{array}{l}\text { Boom } 52014 \text { (herb. van den Boom), } \\
\text { LG DNA } 4239\end{array}$ & van den Boom et al. 2017 & KX459348 \\
\hline Micarea doliiformis & UK & Andersen $178 \mathrm{a}(\mathrm{BG})$ & Schmull et al. 2011 & HQ660557 \\
\hline Micarea elachista & Finland & Launis 67113 , DNA A340 $(\mathrm{H})$ & Launis et al. $2019 b$ & MG707745 \\
\hline Micarea elachista & Sweden & Koffman 399 (herb. Koffman) & Andersen \& Ekman 2005 & AY567755 \\
\hline Micarea elachista & Poland & Czarnota $2986(\mathrm{GPN})$ & Czarnota \& Guzow-Krzemińska 2010 & EF453680 \\
\hline Micarea eximia & Russia & Hermansson 8866b (UPS) & Andersen 2004 & AY756447 \\
\hline Micarea fallax & Czech Republic & Malíček 6127, DNA A608 & Launis et al. 2019a & MK454758 \\
\hline Micarea fallax & Finland & Launis 1010138, DNA A461, H & Launis et al. 2019a & MK454765 \\
\hline Micarea fallax & Finland & Launis 59132, DNA A559 & Launis et al. 2019a & MK454759 \\
\hline Micarea fallax & Belarus & Tsurykau 001c4, DNA A397 & Launis et al. 2019a & MK454760 \\
\hline Micarea fallax & UK, Scotland & Launis 171143, DNA A646, H & Launis et al. 2019a & MK454761 \\
\hline Micarea fallax & Sweden & Svensson 2398, DNA MSv2398, H & Launis et al. 2019a & MK454762 \\
\hline Micarea fallax & Finland & Launis 1710132, DNA A718, H & Launis et al. 2019a & MK454764 \\
\hline Micarea fallax & Finland & Launis 1010139, DNA A453 & Launis et al. 2019a & MK454766 \\
\hline Micarea fallax & Finland & Launis 27122, DNA A440, H & Launis et al. 2019a & MK454763 \\
\hline
\end{tabular}


Table 1. Continued.

\begin{tabular}{|c|c|c|c|c|}
\hline Species & Country/region & Voucher & Reference & $\mathrm{mtSSU}$ \\
\hline Micarea fallax & Finland & Launis 109115 , DNA A605, H & Launis et al. 2019a & MK454757 \\
\hline Micarea fennica & Finland & Launis 3220, DNA A790, (H) & Launis \& Myllys 2019 & MK517716 \\
\hline Micarea fennica & Finland & Launis 68, DNA A117, (H) & Launis \& Myllys 2019 & MK517715 \\
\hline Micarea flagellispora & $\begin{array}{l}\text { Australia, } \\
\text { Tasmania }\end{array}$ & Kantvilas 60/90 (UPS) & Andersen 2004 & AY756448 \\
\hline Micarea flavoleprosa & France & Sérusiaux s.n., DNA 3841, (LG) & Launis et al. 2019a & MK454754 \\
\hline Micarea flavoleprosa & Czech Republic & Malíček 4699, DNA A614, (PRA) & Launis et al. 2019a & MK454755 \\
\hline Micarea flavoleprosa & Czech Republic & Malíček 5098, DNA A616, (H) & Launis et al. 2019a & MK454756 \\
\hline Micarea globulosella & Finland & Launis 67112, DNA A240 (H) & Launis et al. $2019 b$ & MG707743 \\
\hline Micarea globulosella & Finland & Launis 67114, DNA A243 (H) & Launis et al. $2019 b$ & MG707744 \\
\hline Micarea hedlundii & Poland & Czarnota 3915 (GPN) & Czarnota \& Guzow-Krzemińska 2010 & EF453667 \\
\hline Micarea hedlundii & Poland & Czarnota 3895 (GPN) & Czarnota \& Guzow-Krzemińska 2010 & EF453672 \\
\hline Micarea hedlundii & Poland & Czarnota 4589 (GPN) & Czarnota \& Guzow-Krzemińska 2010 & EF453677 \\
\hline Micarea hedlundii & Finland & Launis 67119, DNA A254 $(\mathrm{H})$ & Launis et al. $2019 b$ & MG707749 \\
\hline Micarea hedlundii & Bolivia & AF25384 (KRAM) & Guzow-Krzemińska et al. 2019a & MK561614 \\
\hline Micarea herbarum & Poland & Czarnota 4634 (GPN) & $\begin{array}{l}\text { Czarnota \& Guzow-Krzemińska 2010, } \\
\text { van den Boom et al. } 2017\end{array}$ & EF453692 \\
\hline Micarea herbarum & Netherlands & $\begin{array}{l}\text { P. \& B. van den Boom, 52575, LG } \\
\text { DNA } 4236 \text { (herb. van den Boom) }\end{array}$ & $\begin{array}{l}\text { van den Boom et al. 2017, Launis } \\
\text { et al. } 2019 b\end{array}$ & KX459349 \\
\hline Micarea herbarum & Netherlands & $\begin{array}{l}\text { Brand 63193, LG DNA } 3852 \\
\text { (herb. Brand) }\end{array}$ & van den Boom et al. 2017 & KX459350 \\
\hline Micarea cf. herbarum & Poland & Czarnota 3464 (GPN) & $\begin{array}{l}\text { Czarnota \& Guzow-Krzemińska 2010, } \\
\text { van den Boom et al. } 2017\end{array}$ & EF453665 \\
\hline Micarea incrassata & Norway & Tønsberg 17593 (BG) & Andersen 2004 & AY756449 \\
\hline Micarea isidioprasina & France & Sérusiaux s.n., DNA 3437 (LG) & $\begin{array}{l}\text { van den Boom et al. 2017, Guzow- } \\
\text {-Krzemińska et al. 2019a }\end{array}$ & KX459362 \\
\hline Micarea isidioprasina & Belgium & Sérusiaux s.n., DNA 3609 (LG) & $\begin{array}{l}\text { van den Boom et al. 2017, Guzow- } \\
\text {-Krzemińska et al. } 2019 \text { a }\end{array}$ & KX459363 \\
\hline Micarea isidioprasina & Germany & van den Boom 53248, DNA 4590 (LG) & Guzow-Krzemińska et al. 2019a & MK562030 \\
\hline Micarea isidioprasina & Poland & Kukwa 17367a \& Lubek (UGDA) & Guzow-Krzemińska et al. 2019a & MK562016 \\
\hline Micarea isidioprasina & Poland & Kukwa 17493 (UGDA) & Guzow-Krzemińska et al. 2019a & MK562015 \\
\hline Micarea cf. isidioprasina & USA & Tønsberg 30856 (BG) & $\begin{array}{l}\text { Andersen 2004, Guzow-Krzemińska } \\
\text { et al. } 2019 \text { a }\end{array}$ & AY756452 \\
\hline Micarea laeta & Finland & Launis 49151, DNA A819 (H) & Launis et al. $2019 b$ & MG707772 \\
\hline Micarea laeta & Finland & Launis 59155, DNA A827 (H) & Launis et al. $2019 b$ & MG707774 \\
\hline Micarea laeta & Finland & Launis 49152, DNA A823 (H) & Launis et al. $2019 b$ & MG707775 \\
\hline Micarea laeta & Finland & Launis 1010133 , DNA A477 (H) & Launis et al. $2019 b$ & MG707778 \\
\hline Micarea laeta & Finland & Launis 1010134, DNA A478 (H) & Launis et al. $2019 b$ & MG707779 \\
\hline Micarea laeta & Finland & Launis 1010135, DNA A427 (H) & Launis et al. 2019b & MG707781 \\
\hline Micarea lapillicola & Czech Republic & Printzen s.n. (herb. Printzen) & Andersen \& Ekman 2005 & AY567735 \\
\hline Micarea leprosula & Norway & Andersen 35 (BG) & Andersen \& Ekman 2005 & AY567762 \\
\hline Micarea levicula & Reunion & Sérusiaux s.n., DNA 3532 (LG) & Guzow-Krzemińska et al. 2019a & MK562019 \\
\hline Micarea levicula & Reunion & Sérusiaux s.n., DNA 3585 (LG) & Guzow-Krzemińska et al. 2019a & MK562020 \\
\hline Micarea lignaria & Norway & Andersen $18(\mathrm{BG})$ & Andersen \& Ekman 2005 & AY567748 \\
\hline Micarea lignaria & France & Sérusiaux s.n., DNA 3435 (LG) & van den Boom et al. 2017 & KX459351 \\
\hline Micarea lignaria & Romania & Sérusiaux s.n., DNA 4375 (LG) & van den Boom et al. 2017 & KX459352 \\
\hline Micarea lithinella & Norway & Andersen 80b (BG) & Andersen \& Ekman 2005 & AY567734 \\
\hline Micarea lynceola & Czech Republic & Palice 1.X.1996 (UPS) & Andersen \& Ekman 2005 & AY567738 \\
\hline Micarea magellanica & New Zealand & Malcolm \& Vezda 21.IV.1997 (UPS) & Andersen 2004 & AY756450 \\
\hline Micarea marginata & Bohemia & $\begin{array}{l}\text { Bayerova, Liska \& Palice } 5159 \\
\text { (herb. Palice) }\end{array}$ & Andersen 2004 & AY756451 \\
\hline Micarea melaena & Norway & Andersen $25(\mathrm{BG})$ & Andersen \& Ekman 2005 & AY567743 \\
\hline Micarea melanobola & Finland & Launis 49141, DNA A808, H & Launis et al. 2019a & MK454767 \\
\hline Micarea melanobola & Finland & Launis 266151, DNA A818 & Launis et al. 2019a & MK454773 \\
\hline Micarea melanobola & Finland & Launis 11014, DNA A424, H & Launis et al. 2019a & MK454774 \\
\hline Micarea melanobola & Finland & Launis 79133, DNA A633, H & Launis et al. 2019a & MK454769 \\
\hline Micarea melanobola & Finland & Launis 39151, DNA A817 & Launis et al. 2019a & MK454771 \\
\hline Micarea melanobola & Finland & Launis 286152 , DNA A813 & Launis et al. 2019a & MK454772 \\
\hline Micarea melanobola & Finland & Launis 27123 , DNA A437 & Launis et al. 2019a & MK454770 \\
\hline Micarea meridionalis & Portugal & $\begin{array}{l}\text { van den Boom s.n. (herb. van den } \\
\text { Boom), DNA } 4279 \text { (LG) }\end{array}$ & van den Boom et al. 2017 & KX459353 \\
\hline
\end{tabular}


Table 1. Continued.

\begin{tabular}{|c|c|c|c|c|}
\hline Species & Country/region & Voucher & Reference & $\mathrm{mtSSU}$ \\
\hline Micarea meridionalis & Portugal & $\begin{array}{l}\text { van den Boom s.n. (herb. van den } \\
\text { Boom), LG DNA } 4281\end{array}$ & van den Boom et al. 2017 & KX459354 \\
\hline Micarea meridionalis & Portugal & $\begin{array}{l}\text { van den Boom s.n. (herb. van den } \\
\text { Boom), LG DNA } 4581\end{array}$ & van den Boom et al. 2017 & KX459355 \\
\hline Micarea microareolata & Sweden & Launis 148131, DNA A393 (H) & Launis et al. $2019 b$ & MG707762 \\
\hline Micarea microareolata & Sweden & Launis 148132, DNA A394 (H) & Launis et al. $2019 b$ & MG707763 \\
\hline Micarea microareolata & Finland & Launis 59152, DNA A826 (H) & Launis et al. $2019 b$ & MG707764 \\
\hline Micarea microareolata & Finland & Pykälä 47787, DNA A797 (H) & Launis et al. $2019 \mathrm{~b}$ & MG707765 \\
\hline Micarea microareolata & Finland & Pykälä 47783, DNA A798 (H) & Launis et al. $2019 \mathrm{~b}$ & \\
\hline Micarea microareolata & Finland & Launis 59133, DNA A565 (H) & Launis et al. 2019b & MG707766 \\
\hline Micarea microareolata & Finland & Launis 89133, DNA A629 (H) & Launis et al. $2019 b$ & MG707767 \\
\hline Micarea micrococca & Poland & Czarnota $4456(\mathrm{GPN})$ & Czarnota \& Guzow-Krzemińska 2010 & EF453662 \\
\hline Micarea micrococca & Estonia & Czarnota 4782 (GPN) & Czarnota \& Guzow-Krzemińska 2010 & EF453676 \\
\hline Micarea micrococca & Poland & Czarnota 4553 (GPN) & Czarnota \& Guzow-Krzemińska 2010 & EF453683 \\
\hline Micarea micrococca & Netherlands & $\begin{array}{l}\text { P. \& B. van den Boom } 50314 \\
\text { (herb. van den Boom), LG DNA } 3853\end{array}$ & van den Boom et al. 2017 & KX459356 \\
\hline Micarea micrococca & Netherlands & $\begin{array}{l}\text { P. \& B. van den Boom } 51244 \\
\text { (herb. van den Boom), LG DNA } 3854\end{array}$ & van den Boom et al. 2017 & KX459357 \\
\hline Micarea micrococca & Netherlands & $\begin{array}{l}\text { P. \& B. van den Boom } 52570 \\
\text { (herb. van den Boom), LG DNA } 4237\end{array}$ & van den Boom et al. 2017 & KX459358 \\
\hline Micarea micrococca & Finland & Launis 299101, DNA A100 (H) & Launis et al. $2019 b$ & MG707753 \\
\hline Micarea micrococca & USA & Launis 146127 , DNA A320 (H) & Launis et al. 2019b & MG707754 \\
\hline Micarea micrococca & Netherlands & van den Boom 50316, DNA 3713 (LG) & Guzow-Krzemińska et al. 2019a & MK562023 \\
\hline Micarea microsorediata & Netherlands & van den Boom 50279, DNA 3711 (LG) & Guzow-Krzemińska et al. 2019a & MK562022 \\
\hline Micarea microsorediata & Poland & Kukwa 16994 (UGDA) & Guzow-Krzemińska et al. 2019a & MK562011 \\
\hline Micarea microsorediata & Poland & Kukwa 17053 (UGDA) & Guzow-Krzemińska et al. 2019a & MK562012 \\
\hline Micarea microsorediata & Poland & Kukwa 17546 \& Łubek (UGDA) & Guzow-Krzemińska et al. 2019a & MK562009 \\
\hline Micarea microsorediata & Netherlands & van den Boom 56372 & this paper & MN547362 \\
\hline Micarea minuta & Netherlands & van den Boom 57741 & this paper & MN547360 \\
\hline Micarea misella & Finland & Launis 108111, DNA A264 (H) & Launis et al. $2019 b$ & MG707742 \\
\hline Micarea misella & Norway & Andersen $73(\mathrm{BG})$ & Andersen 2004 & AY567752 \\
\hline Micarea misella & Poland & Czarnota 4593 (GPN) & Czarnota \& Guzow-Krzemińska 2010 & EF453687 \\
\hline Micarea myriocarpa & Norway & Andersen 37 (BG) & Andersen \& Ekman 2005 & AY567736 \\
\hline Micarea nigra & Portugal & van den Boom 53726, DNA 4573 (LG) & Guzow-Krzemińska et al. 2019a & MK562029 \\
\hline Micarea nitschkeana & Czech Republic & Printzen s.n. (herb. Printzen) & Andersen \& Ekman 2005 & AY 567758 \\
\hline Micarea nitschkeana & Poland & Czarnota 3306 (GPN) & Czarnota \& Guzow-Krzemińska 2010 & EF453685 \\
\hline Micarea nowakii & Poland & Czarnota 4181 (GPN) & Czarnota \& Guzow-Krzemińska 2010 & EF453688 \\
\hline Micarea nowakii & Poland & Czarnota 4688 (GPN) & Czarnota \& Guzow-Krzemińska 2010 & EF453689 \\
\hline Micarea nowakii & Romania & Sérusiaux s.n., DNA 4380 (LG) & van den Boom et al. 2017 & KX459359 \\
\hline Micarea nowakii & Romania & Sérusiaux s.n., DNA 4385 (LG) & van den Boom et al. 2017 & KX459360 \\
\hline Micarea nowakii & Finland & Launis 245131, DNA A684 (H) & Launis et al. $2019 b$ & MG707751 \\
\hline Micarea paratropa & Norway & Andersen 94 (BG) & Andersen \& Ekman 2005 & AY 567740 \\
\hline Micarea pauli & Poland & Kukwa 14101 \& Łubek (UGDA) & Guzow-Krzemińska et al. 2019a & MN094374 \\
\hline Micarea pauli & Poland & Kukwa 17240 \& Łubek (UGDA) & Guzow-Krzemińska et al. 2019a & MK562014 \\
\hline Micarea pauli & Poland & Kukwa 17544 \& Łubek (UGDA) & Guzow-Krzemińska et al. 2019a & MK562010 \\
\hline Micarea pauli & Poland & Kukwa 17621 \& Łubek (UGDA) & Guzow-Krzemińska et al. 2019a & MK562013 \\
\hline Micarea peliocarpa & USA & Launis 66123, DNA A324 $(\mathrm{H})$ & Launis et al. $2019 b$ & MG707741 \\
\hline Micarea peliocarpa & Netherlands & $\begin{array}{l}\text { van den Boom } 51318 \text { (herb. van den } \\
\text { Boom) DNA } 3847 \text { (LG) }\end{array}$ & van den Boom et al. 2017 & KX459361 \\
\hline Micarea peliocarpa & Norway & Andersen 29 (BG) & Andersen \& Ekman 2005 & AY567760 \\
\hline Micarea prasina & Russia & Hermansson 4927 (UPS) & $\begin{array}{l}\text { Andersen \& Ekman 2005, Czarnota } \\
\text { \& Guzow-Krzemińska } 2010\end{array}$ & AY567750 \\
\hline Micarea prasina & Poland & Czarnota 3914 (GPN) & Czarnota \& Guzow-Krzemińska 2010 & EF453669 \\
\hline Micarea prasina & Poland & Czarnota 4489 (GPN) & Czarnota \& Guzow-Krzemińska 2010 & EF453678 \\
\hline Micarea prasina & Poland & Czarnota $4319(\mathrm{GPN})$ & Czarnota \& Guzow-Krzemińska 2010 & EF453679 \\
\hline Micarea prasina & Finland & Launis 265101, DNA A92 (H) & Launis et al. $2019 \mathrm{~b}$ & MG707747 \\
\hline Micarea prasina & Finland & Launis 199105, DNA A93 $(\mathrm{H})$ & Launis et al. $2019 b$ & MG707748 \\
\hline Micarea prasina & Germany & van den Boom 50033, DNA 3494 (LG) & Guzow-Krzemińska et al. 2019a & MK562018 \\
\hline Micarea prasina & Germany & van den Boom 50040, DNA 3492 (LG) & Guzow-Krzemińska et al. 2019a & MK562017 \\
\hline Micarea prasina & Poland & Czarnota 3913 (GPN) & Czarnota \& Guzow-Krzemińska 2010 & EF453675 \\
\hline Micarea prasina & UK, Scotland & Launis 171144, DNA A644, H & Launis et al. $2019 \mathrm{a}$ & MK517717 \\
\hline
\end{tabular}


Table 1. Continued.

\begin{tabular}{|c|c|c|c|c|}
\hline Species & Country/region & Voucher & Reference & $\mathrm{mtSSU}$ \\
\hline Micarea prasinella & USA & McCune 25337 (BG) & Andersen \& Ekman 2005 & AY567745 \\
\hline $\begin{array}{l}\text { Micarea pseudomicro- } \\
\text { cocca }\end{array}$ & Finland & Launis 59151, DNA A811 (H) & Launis et al. $2019 \mathrm{~b}$ & MG707755 \\
\hline $\begin{array}{l}\text { Micarea pseudomicro- } \\
\text { cocca }\end{array}$ & Finland & Launis 89132 , DNA A599 $(\mathrm{H})$ & Launis et al. 2019b & MG707756 \\
\hline $\begin{array}{l}\text { Micarea pseudomicro- } \\
\text { cocca }\end{array}$ & Finland & Launis 258131, DNA A603 (H) & Launis et al. $2019 b$ & MG707757 \\
\hline $\begin{array}{l}\text { Micarea pseudomicro- } \\
\text { cocca }\end{array}$ & Scotland & Launis 171141, DNA A645 (H) & Launis et al. $2019 \mathrm{~b}$ & MG707758 \\
\hline Micarea pseudotsugae & Netherlands & van den Boom 58480 & this paper & MN547361 \\
\hline Micarea pusilla & Finland & Launis 1010136, DNA A470, H & Launis et al. 2019a & MK454751 \\
\hline Micarea pusilla & Finland & Launis 1010137, DNA A460 (H) & Launis et al. 2019a & MK454752 \\
\hline Micarea pusilla & Finland & Launis 101035, DNA A464 (H) & Launis et al. 2019a & MK454753 \\
\hline Micarea pycnidiophora & Belgium & Sérusiaux s.n., DNA 3498 (LG) & van den Boom et al. 2017 & KX459364 \\
\hline Micarea pycnidiophora & USA & Tønsberg 30881 (BG) & Andersen \& Ekman 2005 & AY567754 \\
\hline Micarea soralifera & & Kukwa 12722 (UGDA) & Guzow-Krzemińska et al. 2016 & KT119884 \\
\hline Micarea soralifera & Poland & Kukwa 12999 \& Lubek (UGDA) & Guzow-Krzemińska et al. 2016 & KT119885 \\
\hline Micarea soralifera & Poland & Kukwa 13001 \& Lubek (UGDA) & Guzow-Krzemińska et al. 2016 & KT119886 \\
\hline Micarea soralifera & Finland & Launis 1710131, DNA A714 (H) & Launis et al. $2019 b$ & MG707746 \\
\hline Micarea sp. lineage $A$ & UK, Scotland & Launis 171142, DNA A648, (H) & Launis et al. $2019 b$ & MG707782 \\
\hline Micarea $\mathrm{sp}$. & Australia & Kantvilas 466/00 (BG) & Andersen \& Ekman 2005 & AY567757 \\
\hline Micarea squamulosa & Brazil & Caceres and Aptroot 40719 & Hyde et al. 2019 & MK080110 \\
\hline Micarea stipitata & USA & Ekman s. n. (BG) & Andersen \& Ekman 2005 & AY567753 \\
\hline Micarea stipitata & $\begin{array}{l}\text { Canary Islands, } \\
\text { Tenerife }\end{array}$ & Sérusiaux s.n., DNA 3816 (LG) & van den Boom et al. 2017 & KX459365 \\
\hline Micarea subnigrata & UK, Scotland & Coppins 20999 (E) & Andersen 2004 & AY756453 \\
\hline Micarea subviridescens & UK, Scotland & Czarnota 3599 (GPN) & Czarnota \& Guzow-Krzemińska 2010 & EF453666 \\
\hline Micarea synotheoides & Norway & Andersen 47 (BG) & Andersen \& Ekman 2005 & AY567756 \\
\hline Micarea tomentosa & Poland & Czarnota 3949 (GPN) & Czarnota \& Guzow-Krzemińska 2010 & EF453686 \\
\hline Micarea tomentosa & Finland & Launis 11013 , DNA A773 (H) & Launis et al. $2019 \mathrm{~b}$ & MG707750 \\
\hline Micarea turfosa & Norway & Andersen 59 (BG) & Andersen \& Ekman 2005 & AY567742 \\
\hline Micarea usneae & Portugal, Madeira & van den Boom 48057 (BR) & van den Boom \& Ertz 2014 & KF569511 \\
\hline Micarea viridileprosa & Poland & Czarnota 3436 (GPN) & Czarnota \& Guzow-Krzemińska 2010 & EF453671 \\
\hline Micarea viridileprosa & Poland & Czarnota 3869 (GPN) & Czarnota \& Guzow-Krzemińska 2010 & EF453673 \\
\hline Micarea viridileprosa & Poland & Czarnota 4527 (GPN) & Czarnota \& Guzow-Krzemińska 2010 & EF453682 \\
\hline Micarea viridileprosa & Poland & Czarnota $4518(\mathrm{GPN})$ & Czarnota \& Guzow-Krzemińska 2010 & EF453684 \\
\hline Micarea viridileprosa & Netherlands & $\begin{array}{l}\text { P. \& B. van den Boom, 50066, LG } \\
\text { DNA } 3493 \text { (herb. van den Boom) }\end{array}$ & van den Boom et al. 2017 & KX459366 \\
\hline Micarea xanthonica & USA & Tønsberg 25674 (BG) & Andersen 2004 & AY756454 \\
\hline
\end{tabular}

Additionally, several species closely related to the $M$. prasina group belong to this lineage: $M$. adnata, M. elachista, M. globulosella, M. misella, M. pycnidiophora, M. squamulosa and M. synotheoides. The position of $M$. stipitata is not resolved, as two sequences labelled as obtained from this species seem to be unrelated to each other (Fig. 1). Moreover, M. eximia also belongs to this lineage but forms a monophyletic clade together with specimens of $M$. misella (Fig. 1); this needs further study but was beyond the scope of this paper.

The second lineage of Micarea consists of various species which are rather poorly sampled, as most species are represented only by single mtSSU rDNA sequences, with the exception of $M$. denigrata, $M$. doliiformis, $M$. lignaria, M. nitschkeana and $M$. peliocarpa. The newly described $M$. minuta belongs to the $M$. denigrata group and is closely related to $M$. denigrata, $M$. nitschkeana and M. subnigrata (0.98 PP, $76 \mathrm{BS}$ ). Those species form a sister clade to M. lignaria and $M$. magellanica. Moreover, three specimens of $M$. peliocarpa are included here; all belong to the $M$. peliocarpa group (1 PP, $100 \mathrm{BS}$ ) but they are intermingled with other species belonging to this group, which suggests that they may represent different species.

\section{Taxonomy}

Micarea minuta van den Boom, Guzow-Krzemińska \& Kukwa, sp. nov.

(Fig. 2A, C)

MycoBank MB 832479

Diagnosis: Thallus corticolous, granular, dark grey, brownish grey to black, apothecia slightly shiny, $0.05-0.12(-0.15) \mathrm{mm}$ in diam., ascospores ellipsoidal, sometimes slightly curved, (0-)1-septate, 8-9.5 × (2-)2.5-3 $\mu \mathrm{m}$, mesopycnidia often present, semi-immersed ( $\sim 50 \mu \mathrm{m}$ tall, $40-80 \mu \mathrm{m}$ wide), with grey to pale brownish grey tops, mesoconidia shortly bacilliform, $(3.5-4.5 \times 1.0-1.5 \mu \mathrm{m})$, no secondary lichen substances detectable by TLC, Sedifolia-grey pigment present in apothecia (epihymenium). 


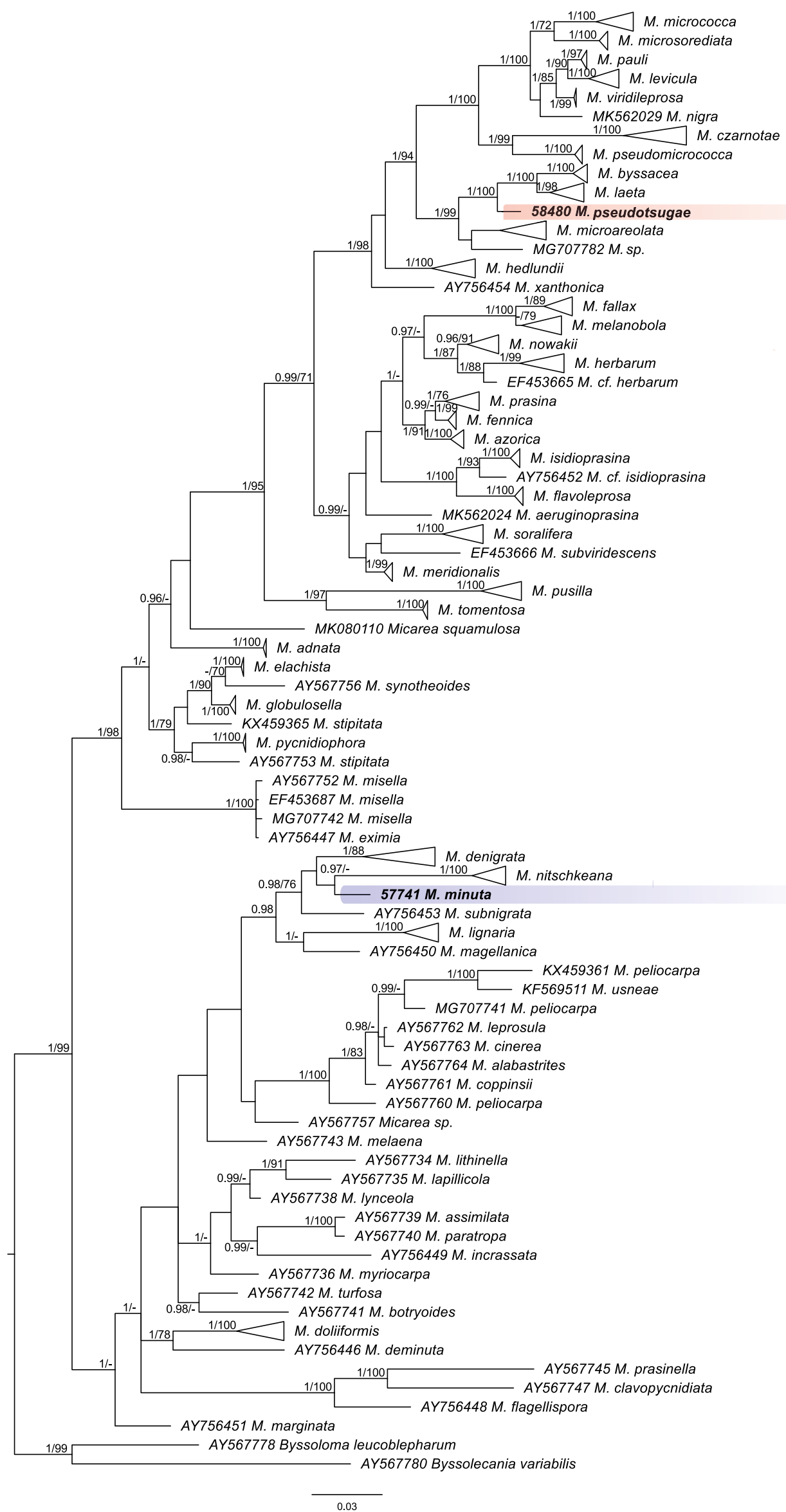

Figure 1. Bayesian tree based on mtSSU rDNA dataset. Posterior probabilities $\geq 0.95$ (first value) in Bayesian analysis and bootstrap supports $\geq 70$ by ML method are indicated near the branches. Highly supported clades with previously described species represented by numerous sequences are collapsed. Herbarium collection numbers for newly sequenced specimens precede the names of species. The newly described species are bolded and shaded. 
Type: The Netherlands, Noord-Brabant Prov., N of Spoordonk, Beverdonkse Dijk, partly unpaved road, roadside trees, Quercus robur along field, $51^{\circ} 32.1^{\prime} \mathrm{N}, 5^{\circ} 16.4^{\prime} \mathrm{E}$. Grid ref. 51.22.23, 5 Oct. 2018, P. \& B. van den Boom 57741 (UGDA - holotype; LG, herb. v.d. Boom - isotype).

Description. Thallus corticolous, consisting of small patches, very thin, continuous to areolate, consisting of small greyish granules; granules irregularly flattened to subglobose, coalescing; upper surface slightly shiny. Cortex without crystals visible in polarized light, pigments lacking. Hypothallus not developed. Apothecia inconspicuous, abundantly present, subglobose, immarginate, sometimes crowded, 0.05-0.12(-0.15) $\mathrm{mm}$ in diam., dark grey, brownish grey to black. Excipulum not developed. Hymenium 25-35 $\mu \mathrm{m}$ high, hyaline. Epithecium spotted dark green-brown. Hypothecium hyaline. Paraphyses abundant, branched and septate, $1.5-2 \mu \mathrm{m}$ wide in middle, tips sometimes widened, up to $3 \mu \mathrm{m}$ and hyaline to rarely brownish. Asci slightly clavate, 8-spored Micarea-type, 16-25 × 7-10 $\mu \mathrm{m}$. Ascospores ellipsoidal, sometimes curved, 8-9.5 × (2-)2.5-3 $\mu \mathrm{m},(0-) 1$-septate, often with an oil droplet in each cell. Mesopycnidia often abundantly present, inconspicuous, grey to pale brownish grey at top, formed out of thallus granules, $\sim 50 \mu \mathrm{m}$ high, $40-80 \mu \mathrm{m}$ wide. Mesoconidia shortly bacilliform, 3.5-4.5 $\times 1.0-1.5 \mu \mathrm{m}$. Micropycnidia or macropycnidia not found.
Photobiont micareoid, 3-8 $\mu \mathrm{m}$ in diam., thin-walled, forming compact clusters.

Chemistry. No lichen secondary substances detected by TLC in thallus and apothecia. Thallus C, K, KC and $\mathrm{Pd}$ negative. Apothecia and pycnidial tops with Sedifolia-grey pigment, $\mathrm{K}+$ violet, $\mathrm{C}+$ violet.

Habitat and distribution. On bark of trunks of Populus sp. and Quercus robur. Accompanying species included mostly crustose lichens (Caloplaca obscurella, Candelariella reflexa, Catillaria nigroclavata, Halecania viridescens, Lecanora barkmaniana) but some macrolichens were present on the same phorophytes (Candelaria concolor, Hyperphyscia adglutinata, Parmotrema perlatum, Physcia clementei, Physconia grisea).

The new species is distributed throughout the south-eastern part of Noord-Brabant Province in the Netherlands, but not yet collected elsewhere. The species is very inconspicuous, even hard to observe under a binocular microscope, and it may be much more widespread than the relatively few collections suggest.

Notes. Morphologically and chemically, Micarea minuta resembles $M$. nitschkeana and $M$. sambuci and looks like a very depauperate specimen of both of them, but these two species have 3-septate ascospores and larger
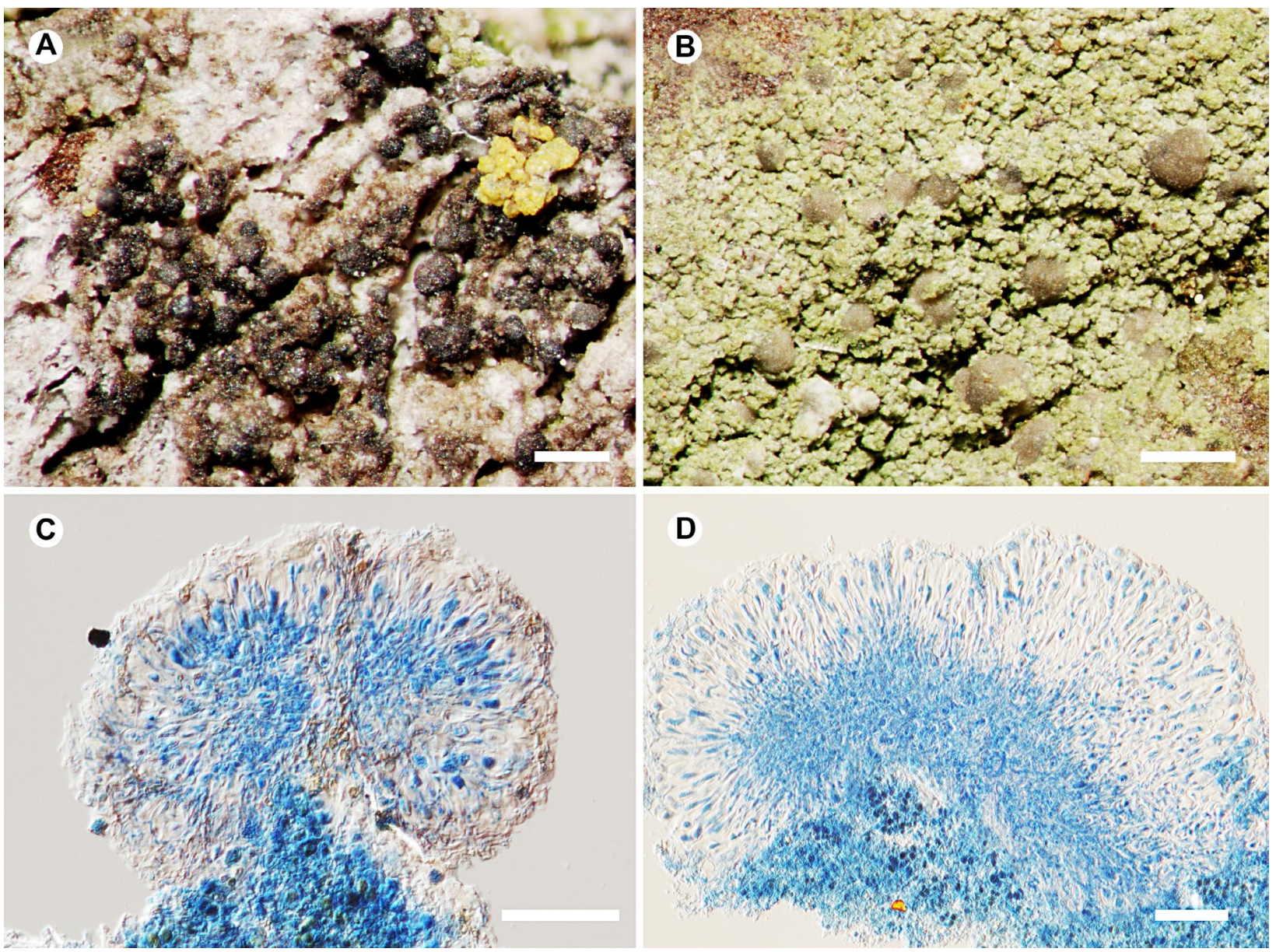

Figure 2. Morphology and sections of apothecia (in lactophenol cotton blue) of the new Micarea species. A, C - M. minuta (holotype); B, D - M. pseudotsugae (holotype). Scales: A $=0.25 \mathrm{~mm} ; \mathrm{B}=0.5 \mathrm{~mm}$; $\mathrm{C}-\mathrm{D}=50 \mu \mathrm{m}$. 
apothecia (0.1-0.25 mm). Mesopycnidia are unknown in $M$. nitschkeana, but in $M$. sambuci they are clearly larger than in the new species and measure 30-150(-250) $\mu \mathrm{m}$ in width (Coppins 1983; Czarnota 2007; van den Boom et al. 2018).

Similar morphology and mostly 1-septate ascospores also characterize $M$. denigrata, but that species has larger apothecia (0.15-0.5 $\mathrm{mm}$ in diam.) and longer ascospores (9-16 $\mu \mathrm{m})$, pycnidia with olivaceous walls $(\mathrm{K}+$ violet $)$, and three types of conidia, and it usually contains gyrophoric acid (Coppins 1983; Czarnota 2007).

The new species can be easily mistaken for the recently described $M$. herbarum, which also has rather small, dark apothecia (0.15-0.25 mm diam.), but that species has different substrate preferences, growing on soft and decaying wood, standing dead trunks, dead and wet stems of herbaceous plants, or directly on soil; moreover, it belongs to the $M$. prasina group. Its mesoconidia tend to be longer, reaching $6 \mu \mathrm{m}$ in length (van den Boom et al. 2017). A species with the same measurements of apothecia $(0.05-0.12 \mathrm{~mm})$ is $M$. hylocomii, but that is a foliicolous lichen with a thin non-granular thallus, few, simple or sparingly branched paraphyses, and a $\mathrm{K}$ and $\mathrm{C}$ negative, light to dark blue-green epihymenium. Also, pycnidia are unknown in this species (Svensson et al. 2017).

Additional specimens examined. THE NETHERLANDS: Noord-Brabant Prov., N of Oirschot, E of Oude Steeg, S of Hoydonck, Beerendonken, edge of meadow with fence posts, group of Populus and small Picea forest, $51^{\circ} 31.5^{\prime} \mathrm{N}, 5^{\circ} 20.1^{\prime} \mathrm{E}$. Grid ref. 51.23.22, on bark of Populus trunk, 14 June 2017, P. \& B. van den Boom 56404 (herb. v.d. Boom); 2 km S of Boxtel, SE of Lennisheuvel, N of Brede Heide, small road (cross-street) with Populus trees along field, $51^{\circ} 34.1^{\prime} \mathrm{N}, 5^{\circ} 19.1^{\prime} \mathrm{E}$. Grid ref. 51.13.31, on bark of Populus trunk, 6 Sept. 2018, P. \& B. van den Boom 57682 (herb. v.d. Boom); S of Liempde, Achterste Broek (N), Smalvelderstraat, mature Populus trees along unpaved road and meadow, $51^{\circ} 33^{\prime} \mathrm{N}, 5^{\circ} 22.9^{\prime} \mathrm{E}$. Grid ref. 51.13.55, on bark of Populus trunk, 27 Oct. 2017, P. \& B. van den Boom 57257 (herb. v.d. Boom).

Micarea pseudotsugae van den Boom, Guzow-Krzemińska \& Kukwa, sp. nov.

(Fig. 2B, D)

Mycobank MB832480

Diagnosis: Thallus areolate, continuous, but at the thallus edge with some separated areoles. areoles consisting of flattened to convex or subsquamulose granules, granules $50-150 \mu \mathrm{m}$ wide, green, apothecia $0.15-0.5 \mathrm{~mm}$ in diam., greyish brown, pale brown to dark brown, adnate to semi-immersed; ascospores cylindric-elliptical, 9-12 × 2.5-5, 0(-1)-septate, mesopycnidia slightly immersed to sessile, white, $50-150(-200) \mu \mathrm{m}$ in diam., top sometimes conical, often gaping, mesoconidia (4.5-)5-6.5 $\times 1.0-1.2 \mu \mathrm{m}$, methoxymicareic acid present.

Type: The Netherlands, Gelderland Prov., Apeldoorn, NW of city, W of Palace Het Loo, small Pseudotsuga forest with trees, up to $1.2 \mathrm{~m}$ in diam., along trail, $52^{\circ} 13.9^{\prime} \mathrm{N}, 5^{\circ} 56.4^{\prime} \mathrm{E}$. Grid ref. 33.13.43, on bark of Pseudotsuga sp., 5 May 2019, P. \& B. van den Boom 58480 (UGDA - holotype; LG, herb. v.d. Boom - isotypes).

Description. Thallus green, thick, rather extensive (up to $5 \mathrm{~cm}$ wide), areolate, continuous, but with some separated areoles at thallus edge. No crystals revealed under polarized light. Areoles consisting of flattened to convex or subsquamulose granules, granules $50-150 \mu \mathrm{m}$ wide. Hypothallus not developed. Apothecia $0.15-0.5 \mathrm{~mm}$ in diam., greyish brown, pale brown to more rarely dark brown (on exposed places sometimes partly greyish), adnate to semi-immersed, first with inconspicuous margin, soon immarginate, flat to slightly convex. Excipulum in young apothecia slightly developed, of thin irregular hyphae. Hymenium 25-35 $\mu \mathrm{m}$ high, hyaline or more rarely with diffuse brownish vertical streaks (if pigment present), with crystals visible under polarized light in hymenium but very sparse in some apothecia. Epihymenium hyaline or very pale brownish. Hypothecium, hyaline. Paraphyses $1.2-1.5 \mu \mathrm{m}$ thick, slightly branched, not widened at apices, rarely slightly brownish at tops. Asci slightly clavate, Micarea-type, 25-32 $\times$ 9-12 $\mu \mathrm{m}, 8$-spored. Ascospores cylindric-elliptical, 9-12 $\times 2.5-5 \mu \mathrm{m}, 0(-1)$-septate, often with 1 or 2 oil droplets. Mesopycnidia often abundantly present, especially if apothecia absent; conspicuous, slightly immersed to sessile, white, 50-150(-200) $\mu \mathrm{m}$ in diam., top sometimes conical, often gaping. Mesoconidia fusiform to bacilliform, (4.5-)5-6.5 × 1.0-1.5 $\mu \mathrm{m}$, with 2 oil droplets. Micropycnidia or macropycnidia not found.

Photobiont micareoid, 4-8 $\mu \mathrm{m}$ in diam., thin-walled, clustered.

Chemistry. Methoxymicareic acid detected by TLC. Thallus does not react with $\mathrm{C}, \mathrm{K}, \mathrm{KC}$ or Pd. Apothecia with Superba-brown pigment ( $\mathrm{K} \pm$ dulling, $\mathrm{C}-, \mathrm{N}+$ becoming slightly orange).

Habitat and distribution. The new species was found so far only on bark or on a stump of Pseudotsuga, especially the bases of trunks in forests poor in lichen species. The only accompanying species in the type locality are Coenogonium pineti, Lepraria incana, L. finkii and Porina leptalea. In the two other localities only Coenogonium pineti has been found as accompanying species.

Micarea pseudotsugae is distributed throughout the central part of the Netherlands to northern Belgium, but not yet collected elsewhere.

Notes. Micarea pseudotsugae is characterized by the areolate, continuous (only at the thallus edge with some separated areoles) thallus, consisting of flattened to convex, often subsquamulose granules, adnate to semi-immersed, mostly pale greyish brown apothecia, crystals (visible in polarized light) present only in the hymenium, conspicuous white mesopycnidia and the presence of methoxymicareic acid. It belongs to the Micarea micrococca group and is most closely related to M. byssacea and M. laeta. It differs from $M$. byssacea mostly by the lack of Sedifolia-grey pigment (Guzow-Krzemińska et al. 2019a; Launis et al. 2019a, b). Micarea laeta has a granular-areolate thallus but the areoles are never subsquamulose, and the thallus is also rarely continuous (Launis et al. $2019 b$ ). Due to the adnate apothecia, the new species is similar to M. microareolata, but the thallus of the latter consists of goniocysts usually coalescing to form convex 
to subglobose small areoles, and its paraphyses are richly branched (Launis et al. 2019b). In addition, in M. laeta and $M$. microareolata small crystalline granules are present in the hymenium and thallus (Launis et al. 2019b), whereas in the new species they are only in the hymenium. The crystals are so sparse in some apothecia that they may seem to be absent at first glance. Other species with similar morphology and the presence of methoxymicareic acid (e.g., M. pseudomicrococca) differ in having convex, rarely adnate and relatively smaller (up to $0.4 \mathrm{~mm}$ diam.) apothecia and non-subsquamulose areoles. Also, some of those (e.g., M. czarnotae) produce Sedifolia-grey pigment or their apothecia are very pale (white to whitish cream) (Launis et al. 2019b).

Micarea pseudotsugae is the second species of the M. micrococca group with crystals (visible in polarized light) only in the hymenium. Micarea czarnotae is the second one, but this species produces Sedifolia-grey pigment in the apothecia (Launis et al. 2019b).

Additional specimens examined. THE NETHERLANDS: Gelderland Prov., Apeldoorn, NW of city, W of Palace Het Loo, forest with mature Fagus, Pinus and Pseudotsuga trees, along trail, $52^{\circ} 14.12^{\prime} \mathrm{N}, 5^{\circ} 55.38^{\prime} \mathrm{E}$. Grid ref. 33.13.32, on bark of Pseudotsuga sp., 26 Nov. 2015, P. \& B. van den Boom 53973 (LG, herb. v.d. Boom); Noord-Brabant Prov., NNE of Vessem, Buikheide, S of Kreielt, forest with Pinus, Betula, Quercus and mature Pseudotsuga trees, $51^{\circ} 26.5^{\prime} \mathrm{N}, 5^{\circ} 18.5^{\prime} \mathrm{E}$. Grid ref. 51.42.25, on bark of Pseudotsuga, 22 March 2019, P. \& B. van den Boom 58108 (herb. v.d. Boom). BELGIUM: Limburg Prov., SW of Molenbeersel, S of Grootbroek, near crossing and bridge over stream, S of chapel, Pseudotsuga forest, $51^{\circ} 09.8^{\prime} \mathrm{N}$, $5^{\circ} 41.9^{\prime}$ E. Grid ref. IFBL C7.35, on stump of Pseudotsuga, 29 Apr. 2004, P. \& B. van den Boom 32644 (herb. v.d. Boom).

\section{Acknowledgements}

We are very grateful to the reviewers for their helpful comments, and to Adam Flakus (Kraków) for help with preparation of some microscopic photographs.

\section{ORCiD}

Pieter P. G. van den Boom

http://orcid.org/0000-0002-1929-2088

Beata Guzow-Krzemińska

http://orcid.org/0000-0003-0805-7987

Martin Kukwa

http://orcid.org/0000-0003-1560-909X

\section{References}

Altschul, S. F., Gish, W., Miller, W., Myers, E. W. \& Lipman, D. J. 1990. Basic Local Alignment Search Tool. Journal of Molecular Biology 215: 403-410.

Andersen, H. L. 2004. Phylogeny and classification of Micarea. Ph.D. thesis, University of Bergen, Norway.

Andersen, H. L. \& Ekman, S. 2005. Disintegration of the Micareaceae (lichenized Ascomycota): a molecular phylogeny based on mitochondrial rDNA sequences. Mycological Research 109: 21-30.

Aptroot, A. \& Cáceres, M. E. D. 2014. New lichen species from termite nests in rainforest in Brazilian Rondonia and adjacent Amazonas. The Lichenologist 46: 365-372.
Brand, A. M., van den Boom, P. P. G. \& Sérusiaux, E. 2014. Unveiling a surprising diversity in the lichen genus Micarea (Pilocarpaceae) in Réunion (Mascarenes archipelago, Indian Ocean). The Lichenologist 46: 413-439.

Castresana, J. 2000. Selection of conserved blocks from multiple alignments for their use in phylogenetic analysis. Molecular Biology and Evolution 17: 540-552.

Coppins, B. J. 1983. A taxonomic study of the lichen genus Micarea in Europe. Bulletin of the British Museum (Natural History), Botany Series 11: 17-214.

Czarnota, P. 2007. The lichen genus Micarea (Lecanorales, Ascomycota) in Poland. Polish Botanical Studies 23: 1-199.

Czarnota, P. \& Guzow-Krzemińska, B. 2010. A phylogenetic study of the Micarea prasina group shows that Micarea micrococca includes three distinct lineages. The Lichenologist 42: 7-21.

Dereeper, A., Guignon, V., Blanc, G., Audic, S., Buffet, S., Chevenet, F., Dufayard, J. F., Guindon, S., Lefort, V., M. Lescot, Claverie, J. M. \& Gascuel, O. 2008. Phylogeny.fr: robust phylogeetic analysis for the non-specialist. Nucleic Acids Research 36: W465-W469.

Guzow-Krzemińska, B. \& Węgrzyn, G. 2000. Potential use of restriction analysis of PCR-amplified DNA fragments in construction of molecular data-based identification keys of lichens. Mycotaxon 76: $305-313$.

Guzow-Krzemińska, B., Czarnota, P., Łubek, A. \& Kukwa, M. 2016. Micarea soralifera sp. nov., a new sorediate species in the M. prasina group. The Lichenologist 48: 161-169.

Guzow-Krzemińska, B., Sérusiaux, E., van den Boom, P. P. G., Brand, A. M., Launis, A., Łubek, A. \& Kukwa, M. 2019a. Understanding the evolution of phenotypical characters in the Micarea prasina group (Pilocarpaceae) and descriptions of six new species within the group. MycoKeys 57: 1-30.

Guzow-Krzemińska, B., Flakus, A., Kosecka, M., Jabłońska, A., Rodriguez-Flakus, P. \& Kukwa, M 2019b. New species and new records of lichens from Bolivia. Phytotaxa 397: 257-279.

Huelsenbeck, J. P. \& Ronquist, F. 2001. MRBAYES: Bayesian inference of phylogenetic trees. Bioinformatics 17: 754-755.

Hyde, K. D., Tennakoon, D. S., Jeewon, R., Bhat, D. J., Maharachchikumbura, S. S. N., Rossi, W., Leonardi, M., Lee, H. B., Mun, H. Y., Houbraken, J., Nguyen, T. T. T., Jeon, S. J., Frisvad, J. C., Wanasinghe, D. N., Lücking, R., Aptroot, A., Caceres, M. E. S., Karunarathna, S. C., Hongsanan, S., Phookamsak, R., Silva, N. I., Thambugala, K. M., Jayawardena, R. S., Senanayake, I. C., Boonmee, S., Chen, J., Luo, Z. L., Phukhamsakda, C., Pereira, O. L., Abreu, V. P., Rosado, A. W. C., Buyck, B., Randrianjohany, E., Hofstetter, V., Gibertoni, T. B., Silva Soares, A. M., Plautz Jr., H. L., Pontes Sotão, H. M., Xavier, W. K. S., Bezerra, J. D. P., Oliveira, T. G. L., Souza-Motta, C. M., Magalhães, O. M. C., Bundhun, D., Harishchandra, D., Manawasinghe, I. S., Dong, W., Zhang, S. N., Bao, D. F., Samarakoon, M. C., Pem, D., Karunarathna, A., Lin, C. G., Yang, J., Perera, R. H., Kumar, V., Huang, S. K., Dayarathne, M. C., Ekanayaka, A. H., Jayasiri, S. C., Xiao, Y. P., Konta, S., Niskanen, T., Liimatainen, K., Dai, Y. C., Ji, X. H., Tian, X. M., Mešić, A., Singh, S. K., Phutthacharoen, K., Cai, L., Sorvongxay, T., Thiyagaraja, V., Norphanphoun, C., Chaiwan, N., Lu, Y. Z., Jiang, H. B., Zhang, J. F., Abeywickrama, P. D., Aluthmuhandiram, J. V. S., Brahmanage, R. S., Zeng, M., Chethana, T., Wei, D. P., Réblová, M., Fournier, J., Nekvindová, J., Barbosa, R. N., Felinto dos Santos, J. E., Oliveira, N. T., Li, G. J., Ertz, D., Shang, Q. J., Phillips, A. J. L., Kuo, C. H., Camporesi, E., Bulgakov, T. S., Lumyong, S., Jones, E. B. G., Chomnunti, P., Gentekaki, E., Bungartz, F., Zeng, X. Y., Fryar, S., Tkalčec, Z., Liang, J. M., Li, G. S., Wen, T. C., Singh, P. N., Gafforov, Y., Promputtha, I., Yasanthika, E., Goonasekara, I. D., Zhao, R. L., Zhao, Q., Kirk, P. M., Liu, J. K., Yan, J. Y., Mortimer, P. E., Xu, J. C. \& Doilom, M. W. 2019. Fungal diversity notes 1036-1150: taxonomic and phylogenetic contributions on genera and species of fungal taxa. Fungal Diversity 96: 1-242.

Kantvilas, G. 2018. Micarea kartana sp. nov. (lichenized Ascomycetes) from Kangaroo Island, South Australia. Swainsona 31: 55-58. 
Landan, G. \& Graur, D. 2008. Local reliability measures from sets of co-optimal multiple sequence alignments. Pacific Symposium on Biocomputing 13: 15-24.

Launis, A. \& Myllys, L. 2019. Micarea fennica, a new lignicolous lichen species from Finland. Phytotaxa 409: 179-188.

Launis, A., Malícek, J., Sérusiaux, E., Svensson, M., Tsurykau, A. \& Myllys, L. 2019a. Sharpening species boundaries in the $\mathrm{Mi}$ carea prasina group with notes on the type species $M$. prasina. Mycologia 111: 574-592.

Launis, A., Pykälä, J., van den Boom, P. P. G., Sérusiaux, E. \& Myllys, L. 2019b. Four new epiphytic species in the Micarea prasina group from Europe. The Lichenologist 51: 7-25.

Miadlikowska, J., Kauff, F., Högnabba, F., Oliver, J. C., Molnár, K., Fraker, E., Gaya, E., Hafellner, J., Hofstetter, V., Gueidan, C., Otálora, M. A. G., Hodkinson, B., Kukwa, M., Lücking, R., Björk, C., Sipman, H. J. M., Burgaz, A. R., Thell, A., Passo, A., Myllys, L., Goward, T., Fernández-Brime, S., Hestmark, G., Lendemer, J., Lumbsch, H. T., Schmull, M., Schoch, C. L., Sérusiaux, E., Maddison, D. R., Arnold, A. E., Lutzoni, F. \& Stenroos, S. 2014. A multigene phylogenetic synthesis for the class Lecanoromycetes (Ascomycota): 1307 fungi representing 1139 infrageneric taxa, 317 genera and 66 families. Molecular Phylogenetics and Evolution 79: $132-168$.

Miller, M. A., Pfeiffer, W., Schwartz, T. 2010. Creating the CIPRES Science Gateway for inference of large phylogenetic trees. Proceedings of the Gateway Computing Environments Workshop (GCE), 14 November 2010, New Orleans, 1-8.

Orange, A., James, P. W. \& White, F. J. 2001. Microchemical methods for the identification of lichens. British Lichen Society, London.

Penn, O., Privman, E., Ashkenazy, H., Landan, G., Graur, D. \& Pupko, T. 2010. GUIDANCE: a web server for assessing alignment confidence scores. Nucleic Acids Research 38 (Web Server issue): W23-W28.

Rambaut, A. 2012 FigTree v1.4.2. http://tree.bio.ed.ac.uk/software/ figtree/

Ronquist, F. \& Huelsenbeck, J. P. 2003. MrBayes 3: Bayesian phylogenetic inference under mixed models. Bioinformatics 19: 1572-1574.
Schmull, M., Miadlikovska, J., Pelzer, M., Stocker-Wörgötter, E., Hoffstetter, V., Franker, E., Hodkingson, B., Reeb V., Kukwa, M., Lumbsch, H. T., Kauff, F. \& Lutzoni, F. 2011 Phylogenetic affiliations of members of the heterogeneous lichen-forming fungi of the genus Lecidea sensu Zahlbruckner (Lecanoromycetes, Ascomycota). Mycologia 103: 983-1003.

Sela, I., Ashkenazy, H., Katoh, K. \& Pupko, T. 2015. GUIDANCE2: accurate detection of unreliable alignment regions accounting for the uncertainty of multiple parameters. Nucleic Acids Research 43 (Web Server issue): W7-W14.

Sérusiaux, E., Brand, A. M., Motiejūnaitè, J., Orange, A. \& Coppins, B. J. 2010. Lecidea doliiformis belongs to Micarea, Catillaria alba to Biatora and Biatora ligni-mollis occurs in western Europe. The Bryologist 113: 333-344.

Stamatakis, A. 2014. RAxML Version 8: A tool for phylogenetic analysis and post-analysis of large phylogenies. Bioinformatics 30 : 1312-1313.

Svensson, M., Ekman, S., Klepsland, J. T., Nordin, A., Thor, G., von Hirschheydt, G., Jonsson, F., Knutsson, T., Lif, M., Spribille, T. \& Westberg, M. 2017. Taxonomic novelties and new records of Fennoscandian crustose lichens. MycoKeys 25: 51-86.

Van den Boom, P. P. G. \& Ertz, D. 2014. A new species of Micarea (Pilocarpaceae) from Madeira growing on Usnea. The Lichenologist 46: 295-301.

Van den Boom, P. P. G., Brand, A. M., Coppins, B. J. \& Sérusiaux, E. 2017a. Two new species in the Micarea prasina group from Western Europe. The Lichenologist 49: 13-25.

Van den Boom, P. P. G., Sipman, H. J. M., Divakar, P. K. \& Ertz, D. 2017 b. New or interesting records of lichens and lichenicolous fungi from Panama, with descriptions of ten new species. Sydowia 69: 47-72.

Van den Boom, P. P. G., Brand, A. M., Coppins, B. J. \& Sérusiaux, E. 2018. A new Micarea species from western Europe, belonging in the Micarea denigrata group. Herzogia 31: 385-389.

Zoller, S., Scheidegger, C. \& Sperisen, C. 1999. PCR primers for the amplification of mitochondrial small subunit ribosomal DNA of lichen-forming ascomycetes. The Lichenologist 31: 511-516. 